\title{
Habermehl: Kommentar ohne Wert
}

\section{Siegwart Lindenberg}

Rijksuniversiteit Groningen, Sociologisch Instituut, Grote Markt 23, NL-9712 HR Groningen. Niederlande

„Gerade weil Durkheims Programm so tief in die moderne soziologische Folklore eingedrungen ist und weil sein substantielles Werk für die Soziologie auch heute noch sehr aktuell ist, neigen viele Soziologen dazu, diese zwei Aspekte nicht zu trennen. Kritik am Programm wird darum leicht als integrale Kritik an Durkheims Werk überhaupt aufgefaßt und mobilisiert zum Teil irrationale $\mathrm{Ab}$ wehrmechanismen" (Lindenberg 1983: 140). Habermehls Kommentar zu meinem Durkheim-Aufsatz (1983) zeigt wieder einmal, daß die Unterscheidung zwischen Programm und substantiellem Werk selbst noch Soziologen schwerfällt, die, wie Habermehl, schon als Studenten gehört haben, wie man solche Unterscheidungen machen muß. Das Resultat ist dementsprechend die soundsovielste "integrale" und irrationale Verteidigung Durkheims.

Gleich zu Beginn seines Kommentars läßt uns Habermehl wissen, daß er aus „Pietät gegenüber dem Klassiker" weder die Argumente von Durkheim noch meine Kritik analysieren will. Statt dessen will er "exemplarisch" ans Werk gehen und nach dem ,ersten besten“ Beispiel greifen. Dieses erste beste Beispiel findet er genau in der Mitte des Artikels. Es geht um meine Behauptung, daß die Eliminierung des Individuums im Widerspruch steht zur Suche nach Ursachen und Folgen. Warum das so ist, wird im zweiten Teil des Artikels behandelt, und ich fasse hier die ausführlichere Argumentation zusammen: allgemeine soziologische Hypothesen sind unvollständig, d. h. wir finden immer wieder (selbst sehr häufig) Situationen, in denen sie nicht zutreffen. Durkheim räumt hier ein, es sei möglich, ,daß die Ursache durch eine entgegengestellte Ursache daran gehindert wurde, ihren Effekt zu produzieren“. Wie können wir nun diese ,entgegengestellten Ursachen“ finden, oder anders gesagt, wie können wir mögliche störende Faktoren theoretisch in den Griff bekommen? Wir müssen dazu explizit ein Niveau in die Theorie aufnehmen, auf dem wir mit guten Gründen mehr Stabilität (d. h. weniger Unvollständigkeit) erwarten können. Dieses Niveau ist die menschliche Natur, die, wenn sie sich überhaupt verändert, sich in jedem Fall im Verhältnis zum Ablauf der Geschichte langsam verändert. Wir können also da- mit rechnen, daß allgemeine Hypothesen auf diesem Niveau weniger unvollständig sind als die soziologischen Hypothesen. Dabei geht es natürlich gerade nicht um „einzigartige“ Individuen, sondern um eine möglichst allgemeine Handlungstheorie. Den meisten Soziologen geht es nicht um Individuen, sondern um soziale Zusammenhänge; daran soll auch die Einbeziehung einer allgemeinen Handlungstheorie nichts ändern. Im Gegenteil, das Argument besagt, daß gerade zur Analyse von sozialen Zusammenhängen eine Einbeziehung des stabileren Niveaus (Handlungstheorie) nötig ist, da wir sonst keine theoretische Heuristik haben, um störende Faktoren zu finden. Durkheim macht selbst immer wieder von dieser Möglichkeit Gebrauch, wie ich durch Beispiele im Artikel angegeben und wie ich ausführlich in einem auch von Habermehl zitierten Artikel dargelegt habe (Lindenberg 1975). Durkheims Programm ist hier (und nicht nur hier) widersprüchlich. Es erlaubt nicht die explizite Einbeziehung von Handlungstheorien (,Eliminierung des Individuums") und fordert doch soziologische Hypothesen ohne Unvollständigkeit (Kausalgesetze). Das Resultat ist bekannt: einerseits gebraucht er doch Handlungstheorien, aber ad hoc, anderseits verwässert er den Begriff des Kausalgesetzes so, daß das Problem der Unvollständigkeit per definitionem verschwindet: „Wann immer wir gezeigt haben, daß zwei Tatsachen in einigen Fällen kovariieren, sind wir sicher, daß wir es mit einer Gesetzmäßigkeit zu tun haben".

Habermehl findet es also aus Gründen der Pietät gar nicht nötig, dies Argument zu rekonstruieren. Stattdessen stellt er zwei Gegenbehauptungen auf: Zum ersten, Durkheims Programm eliminiere Entscheidungsverhalten nicht, lasse also eine Einbeziehung des stabileren Niveaus einer allgemeinen Handlungstheorie explizit in seinem Programm zu. Zum zweiten: Handlungstheorien seien überhaupt nicht nötig, sie seien nur eine (etwas eigenartige) Darstellungsweise von Lindenberg und Konsorten. Diese „Verteidigung" des Durkheimschen Programms ist nicht ohne Ironie. Einmal behauptet er, daß ich („,mit gewollter Blindheit" leugnend) doch mit Durkheim ,an einem Strang“ ziehe; daß Durkheim „weit entfernt davon" sei, das stabilere 
Niveau einer allgemeinen Handlungstheorie programmatisch zu eliminieren. Dann behauptet er, daß Durkheim mit Recht allgemeine Handlungstheorien eliminiere, da kausale Beziehungen auch (oder gerade) ohne diese allgemeine Handlungstheorien aufgestellt werden könnten. Die widersprüchliche Verteidigung des ,integralen“ Durkheims ist typisch und reflektiert die Tatsache, daB Durkheim selbst Handlungstheorien gebraucht, sie aber programmatisch verboten hat.

Habermehl geht mit keinem Wort auf das Problem der Unvollständigkeit ein. Stattdessen unterbaut er die These, daß man für allgemeine soziologische Gesetze keine Handlungstheorien braucht, mit dem Hinweis, daß andere (z. B. Marx) solche Hypothesen ohne Handlungstheorie aufgestellt haben. Ganz nebenbei bemerkt er, daß man „natürlich die empirische Gültigkeit dieser Hypothese(n) in Zweifel ziehen" kann, als hätte die empirische
Gültigkeit nichts mit der Frage zu tun, ob man allgemeine soziologische Hypothesen auch ohne Handlungstheorien aufstellen kann. Das ganze Problem der Unvollständigkeit dreht sich um die empirische Gültigkeit allgemeiner soziologischer Hypothesen. Habermehls Kommentar bleibt weit hinter Durkheims eigenen praktischen (nicht programmatischen) Einsichten zurück.

Weder dem Altmeister noch der Soziologie ist mit so einer „Verteidigung“ geholfen.

\section{Literatur}

Lindenberg, S., 1975: Three psychological theories of a classical sociologist. Mens en Maatschappij 50: 133-153.

Lindenberg, S., 1983: Zur Kritik an Durkheims Programm für die Soziologie. Zeitschrift für Soziologie 12: 139-151. 